\title{
A High Gain Planar Antenna for Wireless Communication in Ism Band
}

\author{
1st P. Venkatesh \\ Department of Electronics and Communication Engineering \\ Ramco Institute of Technology \\ Rajapalayam, India
}

\begin{abstract}
In topical days, the development in communication systems have become indispensable. Hence to meet the system requirements, it is necessary for design of low cost, minimal weight, compact and low profile antennas which are proficient enough to provide and maintain acceptable performance in various aspects. In view of the above context, patch antennas shall be a good participant. So this project focuses the basic study and design of microstrip patch antenna. The motto of this work is to design a microstrip patch antenna for wireless communication application which could operate at $2.45 \mathrm{GHz}$. Computer simulation Technology(CST) microwave studio was here explored to simulate the proposed antenna . While any more antenna designs are supporting the existing systems, inorder to meet requirements of low profile and acceptable gain. Microstrip patch antennas are proposed in this paper. The simulation result based on the essential antenna performance analysis parameters like Return loss, Gain, Radiated power and directivity are discussed. The antennas are designed with a $37 \times 37.6 \times 1.6 \mathrm{~mm}$ and its relative permittivity is 4.3
\end{abstract}

Keywords : UWB, patch antenna, computer simulation technology, Wireless communication

\section{INTRODUCTION}

It is well known that Ultra Wide Band (UWB) technology has become dominant in the past two decades. Due to its available advantages and application this category of band has attracted many academicians and researchers for progress. According to US frequency regulation, an UWB system shall exist between the range of frequency 3.1 to $10.6 \mathrm{GHz}$ [4]. This availability of large frequency range has made students, faculties and researchers to turn their attention towards UWB technology and systems. Moreover, any system that renders a miniscule bandwidth of $500 \mathrm{MHz}$ shall be attributed as an UWB system. An UWB system provides good transmission and reception rate as it is processed using impulse signal. So in order to comply with the ramping claim of high data rate systems, UWB would be an suggestive choice. UWB systems provide a considerable power rating as well. To make an UWB system more effective in its communication the most important key element to be considered is the antenna. An antenna has to be used in the system to transmit and receive the signal. So most of the RF engineers have turned their attention towards designing suitable antenna for efficient transmission and reception of wireless signals through antennas in an UWB systems.

However the task is not so easy as design of antennas have many important and critical parameters to be considered. The situation and scenario of the UWB system where it is going to

\author{
${ }^{2 n d}$ S. Vijayalakshmi \\ Department of Electronics and Communication Engineering \\ R.M.K. Engineering College \\ Chennai, India
}

be used also plays a vital role in the design process[4]. So considering all these points an optimal design has to be done. In olden days antenna with bulk size was considered. But now due to enormous growth of wireless communication systems, antennas with compact size which are highly portable are desired. So the candidate that satisfies the above conditions is the a patch antenna.

\section{MICROSTRIP ANTENNA}

A microstrip patch antenna is a low profile antenna used for variety of applications. It consists of 3 main components namely ground plane, substrate and the patch. The ground plane and patch are made up of metal while the substrate is made up of a dielectric material[7]. The ground plane is placed at the bottom part of the entire material. The substrate is placed above the ground plane and at the top are we have the patch. The patch which is a metal piece of material is the main radiating element in the antenna structure.

In order to obtain and satisfy various properties and radiation characteristics, the shape and the structure of the patch shall be varied or modified. Shapes such as square, rectangle, circle, ellipse etc are some of the shapes that can be used as a patch material. Speaking about performance and radiation characteristics of the antenna, the dimensions of the patch, substrate and the ground plane plays a major role in it. Starting with the patch, if it is rectangular or square, then the length, width and thickness of the patch plays a key role[6]. If the patch is circular or semicircular, then the radius of the patch plays a major role in determining the behavior of the antenna. So it is highly required that, the dimensions have to be carefully calculated and chosen for proper performance of the antenna.

The radiation in a patch antenna happens due to the fringing fields that exists between the two metals. As the metal conductors shall be considered as tow metal plates, the radiation happens between the two metal conducting materials. But as there is substrate between these two metallic contacts, it also affects the performance of the antenna. Now, on considering the substrate material, the things that have to noted down is the thickness of the substrate and the material that is going to be used as a substrate. The choice of material also has a key role in the radiation between the patch and the ground plane[3]. The variable for the thickness of the substrate is $h$ and that for the material is its permittivity $\epsilon_{\mathrm{r}}$. This is called as relative permittivity.

It is now very clear that the radiation happening in a patch antenna is due to the fringing effect between the patch and the 
ground plane. Also more the fringing effect between the patch and the ground plane, better is the radiation from the antenna. So focus has to be done in increasing the fields between the patch and the ground plane. One of the ways to achieve this is by increasing the width of the patch. This shall be done in conjunction with that of the ground plane. To enhance radiation, the value of the relative permittivity of the material shall also be kept low. The thickness of the substrate can also be increased to a considerable extent for increasing the radiation of the patch antenna.

Let us now take a look at the geometry of a simple patch antenna. It is shown as follows.

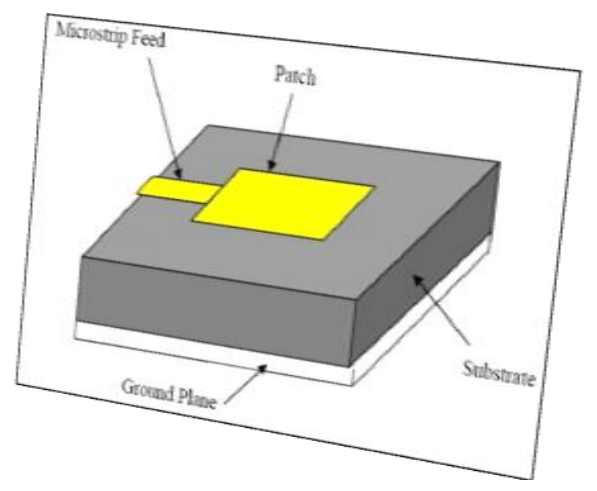

Fig.1. Structure of a Patch Antenna

From the figure1, it is much simpler to understand the arrangement of a microstrip patch antenna. The bottom most part of the antenna is made up of a ground level. This ground level is made up of a metal material. Above the ground region a material called substrate is placed. This is the material which will have naturally some permittivity. Depending upon the application for which the antenna is being designed, the choice of substrate material may vary. Overhead the substrate, there is place the important element called the patch. It is the radiating material in the entire constituent. Another important constituent is the feed. It is the thing that serves as the input connecting line to the patch. in the following section let us look in detail about the feeds used in a patch antenna.

\section{FEEDS}

A patch antenna shall be fed by using the following methods
A. Microstrip line feed
B. Coaxial feed
C. Aperture couple feed

\section{A. Microstrip line feed}

The diagram shown above is an example of line feed. In this type of feed, a microstrip transmission line is used to provide the excitation of the patch. The length of the transmission line is usually chosen to be half the wavelength of the signal.

\section{B. Coaxial feed}

A common technique to feed an patch antenna is the coaxial feed. This type pf feed consists of two coaxial connectors namely the inner connector and outer connector. The inner connector is connected to the and soldered to the patch element. The outer connector is attached to the metallic ground plane. In this type of feed, the feed looks like a point from the top view, which can be moved to our desired location based on the value of the impedance. The coaxial type of feed is shown in the following figure 2 .

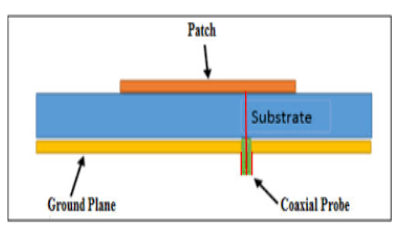

Fig.2. Coaxial probe feed

\section{Aperture couple feed}

In this feeding scheme, instead of using a single substrate, two substrates are used between the ground plane and the patch. The feed line is sandwiched between the two substrates. As we already know that thicker substrates will provide good bandwidth, this type of feeding scheme provides really a good bandwidth as it has two substrates. This type of feeding scheme also reduces spurious radiations. The aperture couple feed is shown in the following figure 3.

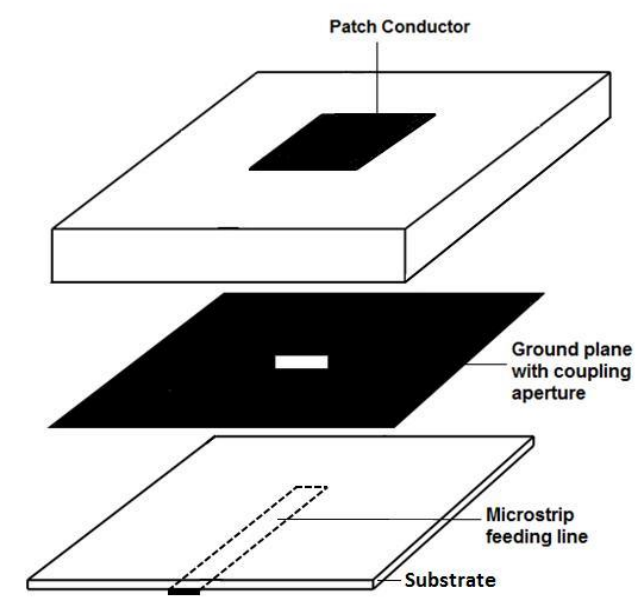

Fig.3.Aperture couple feed

\section{PROPOSED ANTENNA DESIGN}

The proposed antenna is designed for the purpose of communication using the standard IEEE 802.15.6 for UWB wireless communication. The standard can be operated in 3 different bands such as $900 \mathrm{MHz}$ band, $2.3 \mathrm{GHz}$ band and 2.4 $\mathrm{GHz}$ band. As the band of $2.4 \mathrm{GHz}$ has been widely preferred by various products and applications, this paper has been focused in design an appropriate antenna which is capable of operating at $2.45 \mathrm{GHz}$.

As the resonant frequency of the antenna has been fixed, the primary parameters for designing a patch is the length, width and thickness of the patch. By considering the fundamental mode of the antenna, certain unique equations were framed to design a patch. The width of the patch is calculated using the relation

$$
\mathrm{W}=\mathrm{C} /\left(2 \mathrm{f}_{0} \sqrt{ }\left(\mathrm{C}_{\mathrm{r}}+1 / 2\right)\right)
$$


Refer to equation (1), C stands for the velocity of light, $\mathrm{f}_{0}$ is the resonant frequency, $\epsilon_{\mathrm{r}}$ is the relative permittivity of the substrate used which is 4.3 . The substrate used here is FR-4. For all these values, the identified width is $37 \mathrm{~mm}$.

The next step in design is the approximation of length of the patch which is given by the formula

$$
\begin{aligned}
\mathrm{L} & =\frac{c}{2 f 0 \sqrt{\epsilon_{e f f}}} \\
\mathbf{L} & =\mathbf{3 7} \mathbf{~} \mathbf{m m}
\end{aligned}
$$

Similarly the extensive length is given by

$$
\begin{aligned}
& \Delta L=h / \epsilon \text { eff } \\
& \Delta L=0.96 \mathrm{~mm}
\end{aligned}
$$

To calculate the length, the height of the substrate is chosen to be $\mathrm{h}=1.6 \mathrm{~mm}$

\section{RESULTS AND DISCUSSION}

The designed patch antenna is then simulated using Computer Simulation Technology - microwave studio. The following are the parameters were simulated and discussed.

\section{A. Return loss}

It is an important parameter that should be considered while designing an antenna. It measures the reflected energy due to impedance mismatch in an antenna system. For the antenna designed and simulated in this paper, the return loss was found to be $-21 \mathrm{~dB}$. The figure 4 as shown as follows S.Parameters [Magitide in dB]

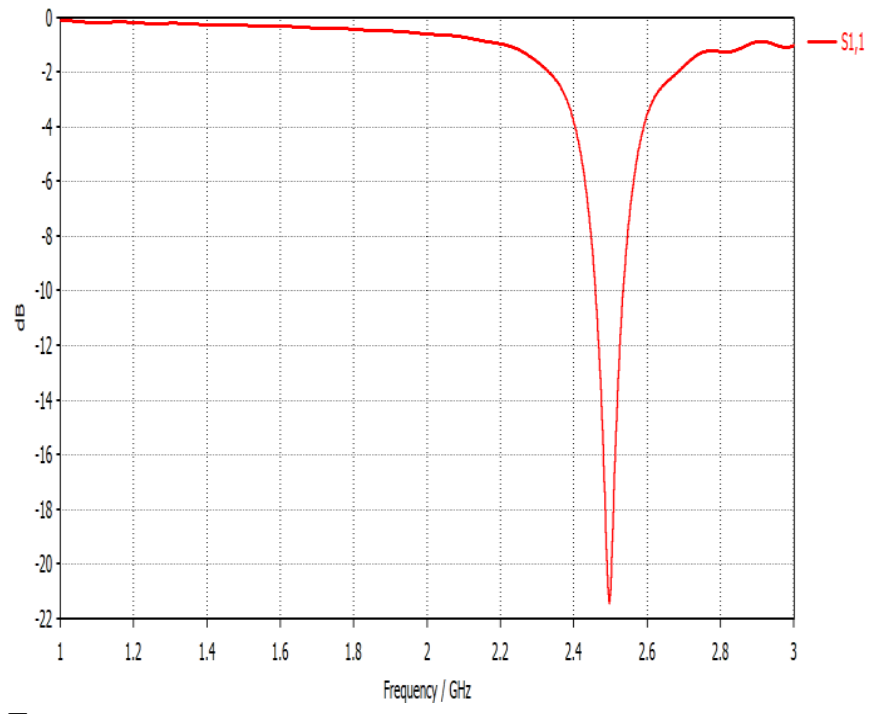

Fig. 4.Return loss

\section{B. VSWR}

It stands for Voltage standing wave ratio. It is the ratio of the wave reflected to the wave incident. For the simulated antenna, the VSWR was found to be 1.19 which is a pretty good value required for an antenna. It shows the antenna has minimum standing wavs in the input port. The simulated figure 5 is shown as follows

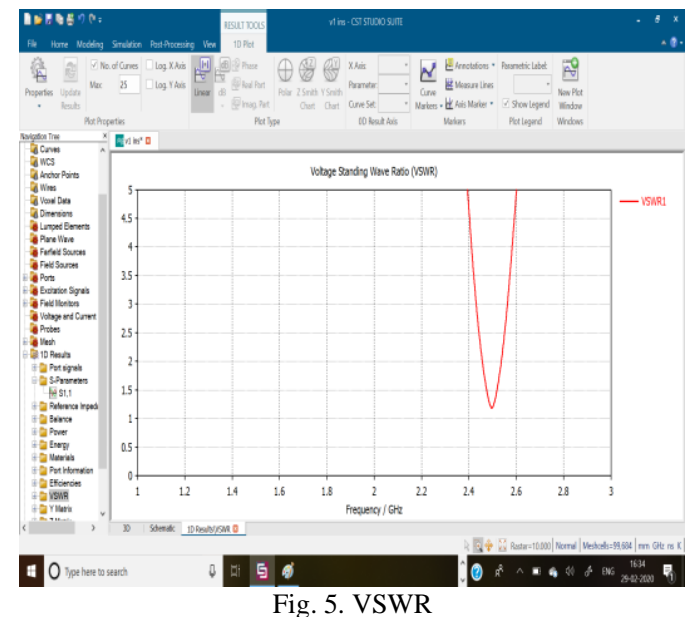

\section{Efficiency}

Efficiency shows how good the antenna is in terms of input and output. The simulated result figure 6 is as follows. The red colored line shows the radiation efficiency and the Green colored line shows the total efficiency. The total efficiency is considered for analyzing the antenna performance as it includes the performance under all classes of loss. It was found that the efficiency is up to $80 \%$.

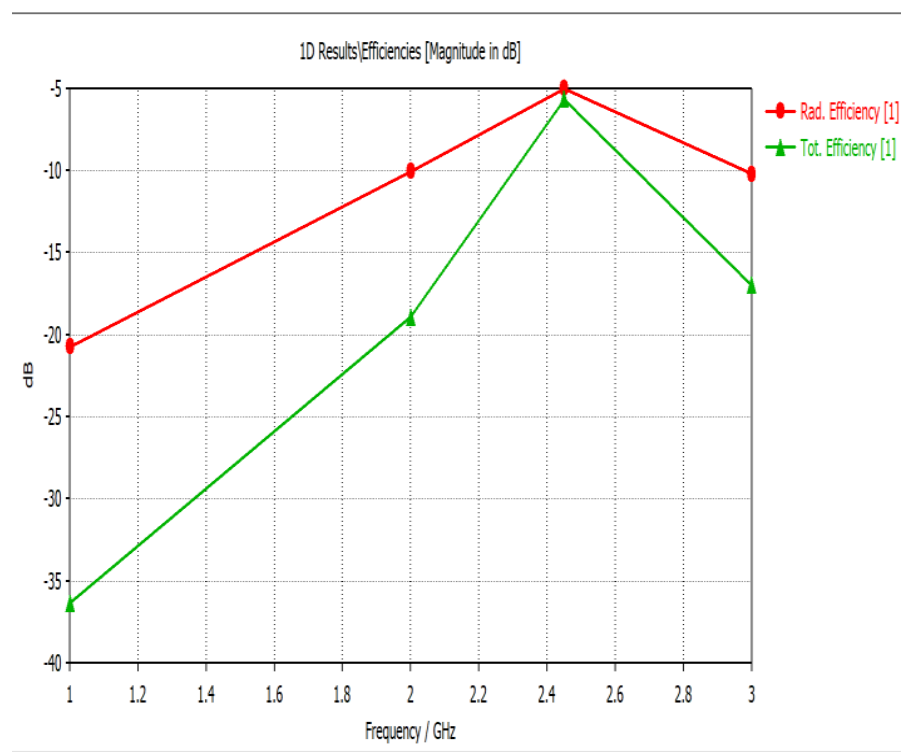

Fig.6. Efficiency

\section{Radiation pattern}

The Figure 7 that shows how an antenna radiates 


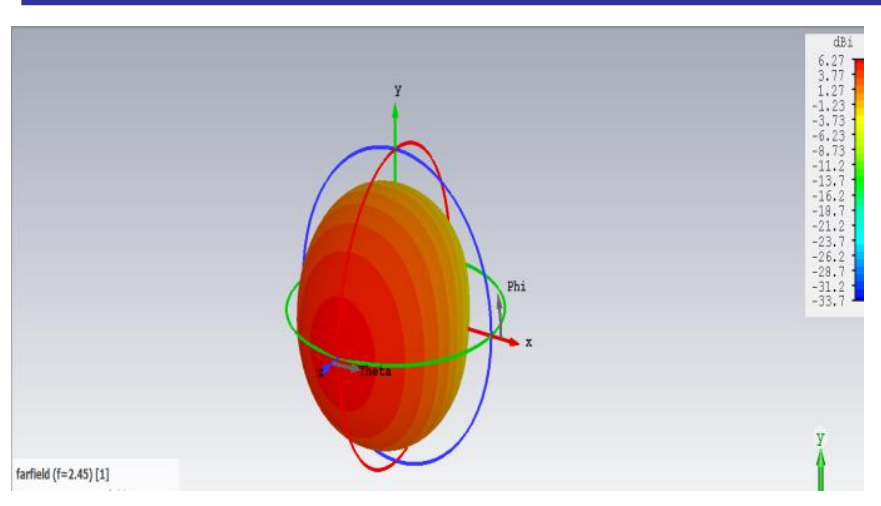

Fig.7. Radiation pattern

From the figure 7 , it is found that at $2.45 \mathrm{GHz}$ the antenna shows a good radiation characteristics. The directivity of the antenna was also found to be around $6 \mathrm{~dB}$. Due to proper impedance matching the return loss of the antenna is also at the required mark. An antenna with good directivity will always show good radiation characteristics. The figure of radiation pattern clearly shows that the radiation from the designed antenna is happening towards the front end of the antenna. This shall be one of the required characteristics for an antenna used in communication

\section{CONCLUSIONS}

In this paper, a suitable microstrip patch antenna for wireless local network was designed. The design was carried out at the most widely used frequency $2.4 \mathrm{GHz}$ range for performing UWB wireless communication. The dimensions of the antenna was chosen and calculated accordingly. From the results of the simulated antenna, it was found that, the antenna provided good radiation characteristics at the desired frequency. The directivity of the antenna was also appreciable. These results were all coinciding with the results that are required for the proper operation of a wireless local network.

\section{REFERENCES}

[1] X. Yang, P. Qin, Y. Liu, Y. Yin and Y. J. Guo, "Analysis and Design of a Broadband Multifeed Tightly Coupled Patch Array Antenna," in IEEE Antennas and Wireless Propagation Letters, vol. 17, no. 2, pp. 217-220, Feb. 2018.

[2] C. Sun, H. Zheng, L. Zhang and Y. Liu, "A Compact Frequency-Reconfigurable Patch Antenna for Beidou (COMPASS) Navigation System," in IEEE Antennas and Wireless Propagation Letters, vol. 13, pp. 967-970, 2014.

[3] Ahmed Shakir A: Design of Rectangular Microstrip Patch Antenna for WLAN and Wi-Max Applications. In: Journal of Engineering and Applied Sciences, vol. 14, pp. 433-438.(2019)

[4] Hans Gregory Schantz,'Introduction to UWB Antennas" in IEEE (UWBST) conference, may 2014.

[5] Ms.Varsharani Mokal, Prof S.R.Gagare, Dr.R.P.Labade, “ Analysis Microstrip Patch Antenna using Coaxial feed and Microstrip line Feed for Wireless Applications" in IORS Journal of Electronics and Communication, Vol. 12. No.3, pp 36-41, June 2017

[6] Ram Krishnan, dr. vijay laxmi,"'Design of Microstrip Antenna for Wireless local Area Network" in International of computer science and mobile computing Vol 4, Issue 4, pp. 361-365, April 2015.

[7] Kai-Fong lee, Kin-Tai Tong Microstrip patch antenna,"Basics and some advances", in proceedings in IEEE, Vol 100, No 7, July 2012.

[8] P.Venkatesh, V. Ranjana, S.Rohini, A. Sangareswari "Design of miniaturized printed antenna for Wi-Fi application", International Journal of Applied Engineering Research, Vol 14., No. 6, 2019.

[9] T.V.Padmavathy, D.S. Bhargava, P.Venkatesh, N.Sivakumar "Design and development of microstrip patch antenna with circular and rectangular slot for structural health monitoring" in Journal of Personal and Ubiquitous Computing, pp. 883-893, April 2018.

[10] I.Linardou, C.Miggliaccio, M. Laheurte "Twin Vivaldi Antenna fed by Coplanar Waveguide" IEEE Electronic Letters, Vol. 33, October 1997.

[11] T.V.Padmavathy, P.Venkatesh, D.S. Bhargava , N.Sivakumar "Design of I-shaped dual C-slotted rectangular microstrip antenna for breast cancer tumor detection" Journal of Cluster Computing, Vol 16, pp 13985-13993, February 2018. 